\title{
Effects of Short-Term Hydroxychloroquine Plus Moxifloxacin Therapy on Corrected QT Interval and Tp-e Interval in Patients With COVID-19
}

\author{
Abdulmecit Afsin ${ }^{\mathrm{a}, \mathrm{d}}$, Kenan Ecemis ${ }^{\mathrm{b}}$, Ramazan Asoglu ${ }^{\mathrm{c}}$
}

\begin{abstract}
Background: Limited data are available regarding hydroxychloroquine (HCQ) and moxifloxacin (MOX) in patients with possible coronavirus disease 2019, (COVID-19). Both drugs may increase risk of malignant ventricular arrhythmias associated with prolongation of QT interval.
\end{abstract}

Methods: A total of 76 subjects with chest tomography findings compatible with COVID-19 pneumonia were enrolled in the study. Standard 12-lead electrocardiogram (ECG) was repeated on days 2 and 5 in patients receiving a combination of $\mathrm{HCQ}+\mathrm{MOX}$. Heart rate, QT interval, Tp-e interval, and Tp-e/QT ratio were measured.

Results: The mean age of the patients was $61.7 \pm 14.8$ years and $54 \%$ had hypertension. Compared to day 2, ECG on day 5 showed significant increases in QT interval $(370.8 \pm 32.5$ vs. $381.0 \pm 29.3$, respectively, $\mathrm{P}=0.001)$, corrected QT (QTc) interval (424 (403 - 436) vs. 442 (420 - 468), respectively, $\mathrm{P}<0.001)$, Tp-e interval (60 (55 $70)$ vs. 65 (57 - 75), respectively, $\mathrm{P}<0.001)$, cTp-e interval (72.2 \pm 12.9 vs. $75.4 \pm 12.7$, respectively, $\mathrm{P}<0.001)$. Moreover, a slight decrease in Tp-e/QT ratio was observed $(0.17 \pm 0.03$ vs. $0.17 \pm 0.02, \mathrm{P}$ $=0.030$ ). QTc was $>500 \mathrm{~ms}$ in $5 \%$ of the patients, and $8 \%$ of patients had an increase in QTc interval $>60 \mathrm{~ms}$. Tp-e/QT ratio was $>0.23$ in $4 \%$ of patients. Five patients died due to pulmonary failure without evidence of ventricular arrhythmia. No ventricular arrhythmia events, including torsades de pointes (TdP), were observed.

Conclusions: HCQ + MOX combination therapy led to increases in QTc interval, Tp-e interval, and cTp-e interval. However, this therapy did not cause ventricular arrhythmia in the short-term observation.

Manuscript submitted July 16, 2020, accepted August 8, 2020

Published online August 15, 2020

a Department of Cardiology, Kahta State Hospital, Adiyaman, Turkey bepartment of Infectious Diseases and Clinical Microbiology, Kahta State Hospital, Adiyaman, Turkey

'Department of Cardiology, Adiyaman Training and Research Hospital, Adiyaman, Turkey

${ }^{\mathrm{d} C}$ Corresponding Author: Abdulmecit Afsin, Department of Cardiology, Kahta State Hospital, Adiyaman, Turkey. Email: abdulmecitafsin@gmail.com

doi: https://doi.org/10.14740/jocmr4288
Keywords: Hydroxychloroquine; Moxifloxacin; QTc interval; cTp-e interval; Ventricular arrhythmia

\section{Introduction}

A pneumonia epidemic, which is considered to have developed due to a new coronavirus, was detected in Wuhan, Hubei province, the People's Republic of China, in 2019, which could not be brought under control and spread around the world within a short time causing a pandemic [1]. The etiological agent has been reported to be novel coronavirus-2019 (2019-nCoV) belonging to the Coronaviridae family, which the World Health Organization (WHO) subsequently named serious acute respiratory syndrome coronavirus 2 (SARS-CoV-2), and the associated disease was named coronavirus disease 2019 (COVID-19) $[2,3]$.

Various agents considered to be effective in the treatment of COVID-19 have been used since the beginning of the pandemic. Chloroquine and hydroxychloroquine (HCQ) are quinine-derived drugs that have long been used in the treatment and prophylaxis of malaria and chronic rheumatic diseases. Chloroquine and HCQ have been reported to be effective in many in vitro experiments against viruses, including SARSCoV-2 [4]. In addition, clinical studies conducted in China demonstrated that chloroquine had marked efficacy against COVID-19-associated pneumonia and acceptable safety [5]. In a small-scale study conducted in France, HCQ was reported to be effective in the treatment of COVID-19, and its efficacy was also suggested to be increased when administered along with azithromycin (AZ) [6]. However, HCQ can extend the QT interval and increase the risk of torsades de pointes (TdP). Moxifloxacin (MOX), from the fluoroquinolone group, is a broad-spectrum antibiotic widely used in atypical pneumonia. MOX has also been reported to extend the QT interval and cause TdP [7].

The QT interval measured on 12-channel electrocardiogram (ECG) shows regional heterogeneity of ventricular repolarization. The time from the peak of the $\mathrm{T}$ wave to the end point (Tp-e) measured on ECG indicates the global dispersion of ventricular repolarization [8]. Studies have shown that increases in both regional and global ventricular repolarization heterogeneity predispose the patient to cardiac arrhythmia [9]. 
Although HCQ and MOX are well-tolerated drugs commonly used in clinical practice, both can cause prolongation of corrected QT (QTc). There are insufficient data in the literature on whether prolongation of QT and Tp-e intervals can occur when HCQ and MOX are used together in the treatment of possible SARS-CoV-2 pneumonia. Drug-induced QT prolongation is an important measure of drug safety as it is associated with increased mortality. This study was performed to evaluate QT and Tp-e intervals, which are ventricular repolarization parameters measured on 12-lead superficial ECG to predict ventricular arrhythmia, in cases of possible COVID-19 with negative results on the polymerase chain reaction (PCR) test receiving a combination of HCQ and MOX.

\section{Materials and Methods}

\section{Study population}

We analyzed the data of patients who presented to our hospital and were diagnosed with suspected COVID-19 between March and April 2020. A total of 76 consecutive subjects with chest computed tomography (CT) findings compatible with COVID-19 pneumonia were enrolled in the study. Diagnosis of COVID-19 was made according to the WHO interim guidelines [10]. HCQ treatment (400 mg twice daily for 1 day, followed by $200 \mathrm{mg}$ twice daily for 4 days) was determined as 5 days according to the guideline published by the Ministry of Health, Republic of Turkey [11] and MOX (400 mg infused once daily for $>5$ days) were started as empirical treatment in patients with a possible diagnosis of COVID-19.

MOX treatment was discontinued in COVID-19 cases confirmed by the PCR test. Therefore, confirmed cases of COVID-19 were excluded from the study. Patients who were using medications that could affect QRS, QT, and Tp-e intervals, including tricyclic antidepressants, antihistamines, and antipsychotics, those with implantable cardioverter-defibrillators, those with a previously known branch or atrioventricular (AV) nodal block, and those with a negative and/or biphasic $\mathrm{T}$ wave on their ECG were also excluded. Those with a heart rate $<60$ beats/min or $>110$ beats/min, and those with congenital long QT syndrome were also excluded. Finally, noncardiac exclusion criteria were pregnancy and breast-feeding.

\section{Study protocol}

All patients presenting to the emergency department or outpatient clinic underwent complete medical assessment, physical examination, laboratory investigation, and $\mathrm{CT}$ of the chest. Epidemiological, clinical, laboratory, and radiological findings were obtained from the electronic medical records and noted in data collection forms.

Blood samples were obtained from the cephalic vein by traumatic venipuncture and mixed with EDTA on hospital admission. Complete white blood cell counts, including neutrophils and lymphocytes, were measured using an automated hematology analyzer (CELL-DYN Ruby; Abbott Diagnostics,
Abbott Park, IL) and expressed as $\times 10^{3}$ cells $/ \mathrm{mm}^{3}$. Hemoglobin and platelet counts were also calculated. Glucose, creatinine, aspartate aminotransferase, alanine aminotransferase, lactate dehydrogenase, bilirubin, sodium, potassium, calcium, ferritin, and C-reactive protein (CRP) levels were analyzed using an Architect c8000 Chemistry System (Abbott Diagnostics) with commercial kits (Abbott Diagnostics). Troponin I and D-dimer assay levels were analyzed using an immunoassay analyzer (AQT90 FLEX; Radiometer, Copenhagen, Denmark).

The chest CT findings were as follows [12]: multiple, patchy, sub-segmental or segmental/lobar ground-glass opacity in unilateral/bilateral lungs; multiple, patchy, or large patches of consolidation in unilateral/bilateral lungs, with slight grid-like or honeycomb-shaped interlobular septal thickening, especially in the middle and lower lobes.

In addition, nasopharyngeal swab samples were collected by professional healthcare personnel in a special sampling room.

The ECG recordings of the patients were acquired from telemetric monitors or a standard 12-lead ECG device. Rhythm recordings were obtained from telemetric monitors (IntelliVue MP5; Philips, Hamburg, Germany) using $25 \mathrm{~mm} / \mathrm{s}$ as the flow rate of the telemetric device and standard derivation (DII derivation) [13].

The 12-lead ECG recordings $(25 \mathrm{~mm} / \mathrm{s}, 10 \mathrm{~mm} / \mathrm{mV})$ were obtained in the supine position using a CardioFax $\mathrm{S}$ device (Nihon Kohden, Tokyo, Japan). Resting heart rate was measured using the ECG data. QT and Tp-e intervals were calculated manually by two cardiologists using the ECG data. Calipers and magnifying glasses were used to reduce measurement errors. The QT interval was calculated as the time from the start of the QRS complex to the end of the T wave. The Tp-e interval was measured as the time from the $\mathrm{T}$ wave peak to the $\mathrm{T}$ wave end point. The measurements were performed on lead II and lead V5, and the longest QT and Tp-e intervals were used for the analyses [14]. The measured values were corrected according to the heart rate using Bazett's formula. QTc interval and corrected Tp-e interval (cTp-e) were obtained, and the ratios of Tp-e/QT and corrected Tp-e/QT (Tp-e/QTc) were calculated. The interobserver and intraobserver variation coefficients for the Tp-e/QT ratio were $2.7 \%$ and $3.1 \%$, respectively, and those for the Tp-e/QTc ratio were $2.7 \%$ and $3.0 \%$, respectively. In addition, the Tisdale score was calculated to evaluate the risk of QTc prolongation. The 12-lead ECG was repeated on days 2 and 5 in patients receiving a combination of HCQ + MOX.

This study was conducted in compliance with the ethical standards of the responsible institution on human subjects as well as with the Helsinki Declaration. Research ethics approval was obtained from the Ethics Committee of Adiyaman University Medical Faculty (approval number: 2020/6-9).

\section{Statistical analysis}

All analyses were performed using SPSS for Windows 15.0 (SPSS Inc., Chicago, IL). Categorical variables are presented as numbers and percentages. Continuous variables were tested for normality of the distribution normality with the Kolmogorov- 
Table 1. Demographic Features and Comorbidities of Patients on Admission ( $\mathrm{N}=76$ )

\begin{tabular}{|c|c|}
\hline Baseline characteristics & $\mathbf{N}(\%)$ \\
\hline \multicolumn{2}{|l|}{ Sex } \\
\hline Female & $44(58 \%)$ \\
\hline Male & $32(42 \%)$ \\
\hline Age (years) & $61.7 \pm 14.8$ \\
\hline Smoking & $11(15 \%)$ \\
\hline \multicolumn{2}{|l|}{ Comorbidities } \\
\hline Heart failure & $13(17 \%)$ \\
\hline Hypertension & $41(54 \%)$ \\
\hline Coronary artery disease & $21(28 \%)$ \\
\hline Cerebrovascular disease & $2(2 \%)$ \\
\hline Diabetes mellitus & $26(34 \%)$ \\
\hline Chronic lung disease $^{\mathrm{a}}$ & $21(28 \%)$ \\
\hline Atrial fibrillation & $2(2 \%)$ \\
\hline Malignancy & $1(1 \%)$ \\
\hline Chronic kidney disease & $5(7 \%)$ \\
\hline Death without cardiac arrhythmia & $5(7 \%)$ \\
\hline
\end{tabular}

${ }^{a}$ Chronic lung disease was defined as chronic obstructive pulmonary disease, asthma, or chronic bronchitis.

Smirnov test. Continuous variables are presented as the mean \pm standard deviation or median (25th -75 th interquartile range) and were compared using the paired $t$-test if the data were normally distributed and Wilcoxon's rank sum test if the data were not normally distributed. Pearson's and Spearman's correlation methods were applied for relationship analysis. In all analyses, $\mathrm{P}<0.05$ was taken to indicate statistical significance.

\section{Results}

A total of 238 patients were eligible for the study. Patients with ECGs that could not be analyzed $(n=10)$, patients with branch block in ECG $(n=5)$, COVID-19 patients with a positive PCR test $(n=47)$, and those without follow-up ECG $(n=40)$ were excluded. In addition, patients with a hospitalization period $<$ 5 days $(n=60)$ were also excluded. The remaining 76 patients were included in the study.

The demographic findings and comorbidities of the study group are shown in Table 1 . The mean age of the patients was $61.7 \pm 14.8$, and $44(58 \%)$ were women. The most common comorbidity in patients was hypertension $(54 \%)$, followed by diabetes mellitus (34\%), and chronic lung disease (28\%). Five patients died during their hospital stay due to pulmonary failure without evidence of ventricular arrhythmia. The laboratory parameters of the patients at the time of presentation are shown in Table 2.

Table 3 presents the ECG parameters of the study group. There was no statistically significant change in heart rate during follow-up. ECG showed statistically significant increases
Table 2. Laboratory Findings of Patients on Admission to Hospital $(\mathrm{N}=76)$

\begin{tabular}{|c|c|}
\hline Hemoglobin, g/dL & $12.9 \pm 1.8$ \\
\hline Platelet count, $\left(\times 10^{3} / \mu \mathrm{L}\right)$ & $215(180-258)$ \\
\hline White blood cell count, $\left(\times 10^{3} / \mu \mathrm{L}\right)$ & $9.1 \pm 3.8$ \\
\hline Neutrophil cell count, $\left(\times 10^{3} / \mu \mathrm{L}\right)$ & $6.4 \pm 3.4$ \\
\hline Lymphocyte cell count, $\left(\times 10^{3} / \mu \mathrm{L}\right)$ & $1.9 \pm 0.9$ \\
\hline Serum creatinine, $\mathrm{mg} / \mathrm{dL}$ & $0.85(0.72-1.07)$ \\
\hline Alanine aminotransferase, $\mathrm{U} / \mathrm{L}$ & $22(16-34)$ \\
\hline Lactate dehydrogenase, $\mathrm{U} / \mathrm{L}$ & $321.6 \pm 114.4$ \\
\hline Serum potassium, $\mathrm{mEq} / \mathrm{L}$ & $4.3 \pm 0.2$ \\
\hline Serum sodium, $\mathrm{mEq} / \mathrm{L}$ & $136.2 \pm 2.4$ \\
\hline C-reactive protein, $\mathrm{mg} / \mathrm{dL}$ & $3.2(0.6-8.5)$ \\
\hline Ferritin, ng/mL & $118(52-220)$ \\
\hline D-dimer, $\mu \mathrm{g} / \mathrm{L}$ & $907(698-1,130)$ \\
\hline Troponin I, ng/mL & $0.01(0.01-0.03)$ \\
\hline Activated partial thromboplastin time, $\mathrm{s}$ & $32.7 \pm 5.0$ \\
\hline Prothrombin time, $\mathrm{s}$ & $1.2 \pm 0.3$ \\
\hline $\mathrm{Ph}$ & $7.37 \pm 0.06$ \\
\hline$\Delta \mathrm{QTc}, \mathrm{ms}$ & $15(3-38)$ \\
\hline Tisdale score & $7.3 \pm 1.1$ \\
\hline Systolic BP, mm Hg & $130.1 \pm 14.1$ \\
\hline Diastolic BP, mm Hg & $80(70-85)$ \\
\hline
\end{tabular}

$\triangle Q T c:$ change in corrected QT interval; BP: blood pressure.

in QT interval (370.8 \pm 32.5 vs. $381.0 \pm 29.3$, respectively, $\mathrm{P}$ $=0.001)$, QTc interval (424 (403 - 436) vs. 442 (420 - 468), respectively, $\mathrm{P}<0.001)$, Tp-e interval (60 (55 - 70) vs. 65 (57 - 75), respectively, $\mathrm{P}<0.001)$, and cTp-e interval (72.2 \pm 12.9 vs. $75.4 \pm 12.7$, respectively, $\mathrm{P}<0.001)$ on day 5 compared to day 2. Furthermore, the ratio of Tp-e/QT $(0.17 \pm 0.03$ vs. 0.17 \pm 0.02 , respectively, $\mathrm{P}=0.030$ ) was decreased significantly from day 2 to day 5 .

In clinical follow-up, four (5\%) patients had QTc $>500$ $\mathrm{ms}$, while $10(8 \%)$ had an increase in QTc interval $>60 \mathrm{~ms}$. Three $(4 \%)$ patients had Tp-e/QT ratio $>0.23$. No atrial arrhythmia and ventricular arrhythmia events, including TdP, were observed in any patient (Table 4). In addition, five patients had nausea without vomiting due to HCQ side effects. Three patients had dizziness due to MOX therapy.

Age and troponin I showed significant positive relations with QTc interval $(\mathrm{r}=0.305, \mathrm{P}=0.007 ; \mathrm{r}=0.318, \mathrm{P}=0.005$, respectively). D-dimer showed also significant positive correlations with cTp-e interval $(\mathrm{r}=0.347, \mathrm{P}=0.002)$ and $\mathrm{Tp}-\mathrm{e} / \mathrm{QTc}$ ratio $(\mathrm{r}=0.339, \mathrm{P}=0.003)$. The correlations between $\mathrm{ECG}$ and clinical parameters are presented in Table 5.

\section{Discussion}

We evaluated the ECG parameters showing heterogeneity 
Table 3. Electrocardiographic Parameters of the Study Group $(\mathrm{N}=76)$

\begin{tabular}{llll} 
& 2nd day & 5th day & P \\
\hline Heart rate (beats/min) & $78(72-90)$ & $80(73-90)$ & 0.127 \\
QT interval (ms) & $370.8 \pm 32.5$ & $381.0 \pm 29.3$ & 0.001 \\
QTc interval (ms) & $424(403-436)$ & $442(420-468)$ & $<0.001$ \\
Tp-e interval (ms) & $60(55-70)$ & $65(57-75)$ & $<0.001$ \\
cTp-e interval (ms) & $72.2 \pm 12.9$ & $75.4 \pm 12.7$ & $<0.001$ \\
Tp-e/QT ratio & $0.17 \pm 0.03$ & $0.17 \pm 0.02$ & 0.03 \\
Tp-e/QTc ratio & $0.17 \pm 0.03$ & $0.17 \pm 0.03$ & 0.228 \\
\hline
\end{tabular}

QTc: corrected QT; Tp-e: transmural dispersion of repolarization; cTp-e: corrected transmural dispersion of repolarization.

Table 4. Increased Ventricular Arrhythmia Risk and Adverse Events in Study Population $(\mathrm{N}=76)$

\begin{tabular}{llll} 
& N & \% & Mean \pm SD \\
\hline 5th day with QTc interval $>500 \mathrm{~ms}$ & 4 & 5 & $516.3 \pm 13.2$ \\
Increase in the QTc interval of $>60 \mathrm{~ms}$ & 10 & 8 & $70.4 \pm 12.4$ \\
Tp-e interval $\geq 110 \mathrm{~ms}$ & 0 & 0 & - \\
cTp-e interval $\geq 110 \mathrm{~ms}$ & 0 & 0 & - \\
Tp-e/QT ratio $>0.23$ & 3 & 4 & $0.24 \pm 0.07$ \\
Tp-e/QTc ratio $>0.23$ & 0 & 0 & - \\
Atrial arrhythmia & 0 & 0 & - \\
Torsades de pointes & 0 & 0 & - \\
Nonsustained ventricular tachycardia & 0 & 0 & - \\
Sustained ventricular tachycardia & 0 & 0 & - \\
\hline
\end{tabular}

QTc: corrected QT; Tp-e: transmural dispersion of repolarization; cTp-e: corrected transmural dispersion of repolarization; SD: standard deviation.

of ventricular repolarization in patients with possible COVID-19 pneumonia receiving HCQ + MOX therapy. Our results showed that QTc increased from a mean baseline of $424 \mathrm{~ms}$ to a maximum of $442 \mathrm{~ms}$ after 5 days, with approximately $5 \%$ of the patients developing QTc $>500 \mathrm{~ms}$. There was also increase in the cTp-e interval from $72 \mathrm{~ms}$ to $75 \mathrm{~ms}$. No ventricular arrhythmia and atrial arrhythmia events were observed.

Long QT syndromes are cardiac repolarization disorders characterized by prolonged QT interval on ECG. These repolarization disorders can lead to rapid polymorphic ventricular tachycardia, known as TdP, syncope, or sudden cardiac death. MOX, a member of the fluoroquinolone group, is now widely used in treatment of atypical pneumonia and can result in acquired long QT syndrome. MOX blocks the rapid activating delayed rectifier potassium current (IKr) encoded by the human ether-a-go-go-related gene $(H E R G)$ in a dose-dependent

Table 5. Correlations Between the Fifth Day Electrocardiography and Clinical Parameters

\begin{tabular}{|c|c|c|c|c|c|c|}
\hline & & iterval & & terval & & Tc ratio \\
\hline & $\mathbf{r}$ & $\mathbf{P}$ & $\mathbf{r}$ & $\mathbf{P}$ & $\mathbf{r}$ & $\mathbf{P}$ \\
\hline Age, (years) & 0.305 & $0.007 *$ & -0.118 & 0.310 & -0.003 & 0.978 \\
\hline Neutrophil, $10^{3} / \mu \mathrm{L}$ & 0.013 & 0.914 & -0.080 & 0.492 & -0.094 & 0.418 \\
\hline Lymphocyte, $10^{3} / \mu \mathrm{L}$ & -0.062 & 0.594 & -0.053 & 0.648 & -0.038 & 0.745 \\
\hline D-dimer, $\mu \mathrm{g} / \mathrm{L}$ & 0.213 & 0.064 & 0.347 & $0.002 *$ & 0.339 & $0.003^{*}$ \\
\hline Troponin I, ng/mL & 0.318 & $0.005^{*}$ & 0.106 & 0.441 & -0.079 & 0.567 \\
\hline $\mathrm{CRP}, \mathrm{mg} / \mathrm{L}$ & 0.016 & 0.891 & 0.001 & 0.991 & 0.002 & 0.985 \\
\hline
\end{tabular}

${ }^{*} \mathrm{P}<0.05$. WBC: white blood cell count; CRP: C-reactive protein. 
manner, thereby causing the prolongation of the QT interval and TdP [15].

It has been known for many years that HCQ has antiviral activity. There have been a number of studies on the effectiveness of both HCQ alone and HCQ + AZ combination in COVID-19. Discussion continues in the medical community regarding the effectiveness of HCQ in the treatment of COVID-19. In vitro and preliminary clinical studies have shown that the use of HCQ alone or in combination with AZ is useful in the treatment of SARS-CoV-2. However, these clinical trials usually consisted of a small number of patients $[6,16]$. In a study including 1,061 patients, Million et al [17] reported that the combination of HCQ and AZ was safe to administer in patients before the development of COVID-19 complications and was associated with a very low mortality rate. In contrast, other groups have stated that HCQ is not effective in the treatment of COVID-19. For example, in an observational study in 1,367 patients with COVID-19, Geleris et al reported that HCQ administration was not associated with either lowered or increased risk of the composite end point of intubation or death [18].

Due to its immunomodulatory characteristics, HCQ has been used for a long time in treatment of autoimmune diseases, such as rheumatoid arthritis and systemic lupus erythematosus, and its cardiovascular safety is good [19]. Moreover, information about cardiac side effects of HCQ is limited to a few case reports. Adverse events, such as prolonged QRS, prolonged QT interval, TdP, and ventricular arrhythmia, have been observed in individuals receiving HCQ due to autoimmune diseases $[20,21]$. Chloroquine causes prolonged QT by increasing the ventricular myocardial action potential, by affecting $\mathrm{Na}^{+}$and $\mathrm{Ca}^{2+}$ channels involved in depolarization, $\mathrm{IKr}$ channels involved in repolarization and, especially, inward rectifier $\mathrm{K}^{+}$current (IK1 current), which stabilizes the resting membrane potential $[15,22]$. The value of the QT interval for predicting TdP risk is low, but risk is known to be greater when QT > $550 \mathrm{~ms}$ [23]. However, case reports and small patient series with drug-induced TdP have shown that QTc exceeding the $500 \mathrm{~ms}$ threshold or $>60 \mathrm{~ms}$ prolongation in QTc compared to baseline ECG are associated with increased cardiac risk [24]. Therefore, when using two or more drugs that prolong QTc in combination, in the treatment of COVID-19, there are concerns about cardiac arrhythmia and sudden cardiac death related to prolonged QT.

The preliminary safety results of a randomized, doubleblind, phase IIb clinical trial (CloroCovid-19 Study) comparing the use of chloroquine at a high dose (a total dose of 12,000 $\mathrm{mg}$ over 10 days) and a low dose (a total dose of 2,700 mg over 5 days) in the treatment of SARS-CoV-2 have been published. The outcome of the first 81 patients in the high-dose chloroquine arm showed a higher rate of QTc $>500 \mathrm{~ms}(25 \%)$ and a trend toward higher lethality (17\%) compared to those given the lower dose [25]. Therefore, the researchers stopped enrolling patients in the high-dose chloroquine arm. In addition, HCQ is less toxic than chloroquine and, according to in vitro studies, the former is three times more potent than the latter [26].

There have been a number of clinical studies to address the safety of the HCQ + AZ combination in COVID-19 pa- tients, and no serious cardiac toxicity was observed in these studies [27-30]. In a clinical study of 84 COVID-19 patients treated with a combination of $\mathrm{HCQ}+\mathrm{AZ}$, the results indicated that $11 \%$ of patients had QTc $>500 \mathrm{~ms}$ and new development of acute renal failure was a strong predictor of extreme QT prolongation, while the baseline QTc was not a predictor of severe QTc prolongation [27]. In a study of 201 patients, Saleh et al [28] reported that the maximum QTc was $470 \mathrm{~ms}$ in patients who received combined HCQ + AZ treatment and $453 \mathrm{~ms}$ in those undergoing monotherapy. The authors did not observe any ventricular arrhythmia. In a population of 90 patients who tested positive for COVID-19 by PCR, Mercuro et al [29] treated 37 patients with HCQ and 53 patients with the combination of HCQ + AZ. Seven of the 37 patients receiving HCQ alone and 11 of the 53 patients receiving combination therapy had prolonged QTc $\geq 500 \mathrm{~ms}$. Furthermore, one patient who received the combination therapy developed TdP. In a study performed in 40 patients with a positive PCR test, Bessiere et al [30] reported that the number of patients with QTc $\geq 500 \mathrm{~ms}$ was higher in the HCQ + AZ combination group, but they did not detect any ventricular arrhythmia.

In this study, while only four cases had QTc interval > $500 \mathrm{~ms}, 10$ patients had prolongation of QTc $>60 \mathrm{~ms}$ in comparison with the previous ECG. In addition, no ventricular arrhythmia, including Tdp, was observed during the follow-up of these patients. We observed a similar risk of arrhythmia in patients with a positive PCR test who received HCQ + AZ combination therapy compared to several previous studies. The absence of malignant ventricular arrhythmias may have played a major role in the short duration of treatment. Our study showed that this combination therapy can be administered safely in COVID-19 patients with close ECG monitoring. Randomized controlled trials are needed to investigate the effects of $\mathrm{HCQ}+$ MOX combination on QT interval.

Numerous experimental and clinical studies have reported the poor performance of QT interval for demonstrating proarrhythmic sensitivity $[31,32]$. As the T wave shows ventricular repolarization on ECG, studies have also investigated the relationship between drug-induced arrhythmia and changes in $\mathrm{T}$ wave morphology. It has been reported that ECG parameters, such as the Tp-e interval and Tp-e/QT ratio, reflect the transmural dispersion of repolarization and show better repolarization heterogeneity than the QT interval. It has been reported that increases in Tp-e interval and Tp-e/QT ratio indicate increased dispersion of repolarization, which is predictive of ventricular arrhythmia and cardiovascular death $[8,33]$. In addition, Tp-e and QT interval have been reported to be affected by body mass index and heart rate, while the $\mathrm{Tp}$-e/QT ratio is unaffected by these factors, and it is more sensitive for predicting ventricular arrhythmia [34]. While the normal range of Tp-e interval is $40-110 \mathrm{~ms}$, that of the Tp-e/QT ratio has been reported to be $0.17-0.23[35,36]$. In a recent meta-analysis, Tse et al [35] reported that the Tp-e intervals were longer and the Tp-e/QT ratios were higher in patients showing adverse events, such as Tdp, than in those without such events among those with acquired QT prolongation. In subgroup analysis of the study, they found that the Tp-e interval was $149 \pm 16 \mathrm{~ms}$ and the Tp-e/QT ratio was 
$0.27 \pm 0.05$ in patients who developed cardiac events in the drug-induced long QT syndrome. Topilski et al [36] reported that Tp-e interval $\geq 117 \mathrm{~ms}$ was associated with ventricular arrhythmia. Yamaguchi et al [37] determined that in patients with acquired long QT syndrome, Tp-e/QT ratio $>0.28$ increased the risk of TdP.

The effects of HCQ on Tp-e interval and Tp-e/QT ratio remain unclear. Taubel et al [38] reported that MOX caused prolongation of Tp-e interval. In the present study, we found that patients had increased Tp-e and cTp-e intervals and Tp-e/ QT ratio during follow-up. On day 5 of follow-up, none of the patients had Tp-e or cTp-e interval $\geq 110 \mathrm{~ms}$, and the increases in these intervals were within the respective normal range. Three patients had $\mathrm{Tp}-\mathrm{e} / \mathrm{QT}$ ratio $>0.23$, and the $\mathrm{Tp}-\mathrm{e} /$ QT ratio of these patients increased only slightly. There were no patients with cTp-e/QT ratio $>0.23$. Our results showed that short-term doses ( 5 days) of HCQ + MOX combination therapy were accompanied by slight increases in Tp-e interval and cTp-e interval, which were not related to ventricular arrhythmia. In addition, the evaluation of these parameters with QTc may be more valuable in predicting drug-induced ventricular arrhythmia. Randomized controlled studies are required to validate the results and determine the cut-off values for Tp-e interval and Tp-e/QT ratio in predicting drug-induced ventricular arrhythmia.

There have been a number of reports regarding the monitoring and management of QT prolongation caused by HCQ in SARS-CoV-2. Some groups have suggested that a baseline ECG should be performed in all patients and repeat ECGs should be performed during the period of hospitalization, while others recommend ECG monitoring only in high-risk patients [39]. Our study showed that ECG monitoring once or twice is sufficient.

This study has several limitations. This was a retrospective and observational study conducted in a single center with a small number of patients. Use of a Holter monitor for follow-up of QTc and cTp-e intervals might have been useful in determining cardiac arrhythmia in these patients. However, the risk of COVID-19 complicates this procedure. Duration of the combination therapy was limited to 5 days in this study. However, longer combination therapy is more useful to determine its adverse effects. Tisdale score was calculated based on the ECG results from day 2 and not the basal ECG.

\section{Conclusions}

The results of this study showed that in possible COVID-19 pneumonia patients, HCQ + MOX combination therapy led to increases in QTc and cTp-e intervals; however, short-term HCQ + MOX combination therapy did not cause ventricular arrhythmia. Nevertheless, the possibility that dysrhythmic activity may increase synergistically with the use of this drug combination should always be taken into consideration. To evaluate the risk of ventricular arrhythmia in the treatment of COVID-19 patients, it may be safer and more appropriate to evaluate the QTc and cTp-e intervals together with the Tp-e/ QTc ratio. Due to the risk of infection, it may be sufficient to perform ECG monitoring on days 2 and 5 rather than daily during the follow-up of COVID-19 patients.

\section{Acknowledgments}

None to declare.

\section{Financial Disclosure}

None to declare.

\section{Conflict of Interest}

The authors declare no potential conflict of interest.

\section{Informed Consent}

Not applicable.

\section{Author Contributions}

AA contributed to conceptualization, analysis, writing, and editing; KE contributed to literature search and critical editing; RA contributed to data curation, analysis, supervision and resources.

\section{Data Availability}

The data supporting the findings of this study are available from the corresponding author upon reasonable request.

\section{References}

1. Jin Y, Yang H, Ji W, Wu W, Chen S, Zhang W, Duan G. Virology, epidemiology, pathogenesis, and control of COVID-19. Viruses. 2020;12(4).

2. Lai CC, Shih TP, Ko WC, Tang HJ, Hsueh PR. Severe acute respiratory syndrome coronavirus 2 (SARSCoV-2) and coronavirus disease-2019 (COVID-19): The epidemic and the challenges. Int J Antimicrob Agents. 2020;55(3):105924.

3. Gorbalenya AE, Baker SC, Baric RS, de Groot RJ, Drosten C, Gulyaeva AA, Haagmans BL, et al. The species Severe acute respiratory syndrome-related coronavirus: classifying 2019-nCoV and naming it SARS-CoV-2. Nat Microbiol. 2020;5(4):536-544.

4. Liu J, Cao R, Xu M, Wang X, Zhang H, Hu H, Li Y, et al. Hydroxychloroquine, a less toxic derivative of chloroquine, is effective in inhibiting SARS-CoV-2 infection in vitro. Cell Discov. 2020;6:16.

5. Gao J, Tian Z, Yang X. Breakthrough: Chloroquine phos- 
phate has shown apparent efficacy in treatment of COVID-19 associated pneumonia in clinical studies. Biosci Trends. 2020;14(1):72-73.

6. Gautret P, Lagier JC, Parola P, Hoang VT, Meddeb L, Mailhe M, Doudier B, et al. Hydroxychloroquine and azithromycin as a treatment of COVID-19: results of an open-label non-randomized clinical trial. Int J Antimicrob Agents. 2020;56(1):105949.

7. Koide T, Shiba M, Tanaka K, Muramatsu M, Ishida S, Kondo Y, Watanabe K. Severe QT interval prolongation associated with moxifloxacin: a case report. Cases J. 2008;1(1):409.

8. Kors JA, Ritsema van Eck HJ, van Herpen G. The meaning of the Tp-Te interval and its diagnostic value. J Electrocardiol. 2008;41(6):575-580.

9. Sauer A, Wilcox JE, Andrei AC, Passman R, Goldberger JJ, Shah SJ. Diastolic electromechanical coupling: association of the ECG T-peak to T-end interval with echocardiographic markers of diastolic dysfunction. Circ Arrhythm Electrophysiol. 2012;5(3):537-543.

10. Clinical management of severe acute respiratory infection when novel coronavirus ( $\mathrm{nCoV}$ ) infection is suspected. https://www.who.int/publications-detail/clinical-management-of-severe-acute-respiratory-infection-whennovel-coronavirus-(ncov)-infection-is-suspected. Accessed May 27, 2020.

11. T.C. Saglik Bakanligi Halk Sagligi Genel Mudurlugu. COVID-19 (SARS-CoV-2 Enfeksiyonu Rehberi, Bilim Kurulu Calismasi [online]. 2020. Website: https://covid19bilgi.saglik.gov.tr/depo/rehberler/COVID-19 Rehberi. pdf. Accessed April 14, 2020.

12. Liu J, Liu S. The management of coronavirus disease 2019 (COVID-19). J Med Virol. 2020.

13. Ventricular arrhythmia risk due to hydroxychloroquineazithromycin treatment for COVID-19 - American College of Cardiology. Available at: https:/www.acc.org/ latest-in-cardiology/articles/2020/03/27/14/00/ventricular-arrhythmia-risk-due-to-hydroxychloroquine- azithromycin-treatment-for-covid-19. Accessed Apr 28, 2020.

14. Sapp JL, Alqarawi W, MacIntyre CJ, Tadros R, Steinberg C, Roberts JD, Laksman Z, et al. Guidance on minimizing risk of drug-induced ventricular arrhythmia during treatment of COVID-19: a statement from the canadian heart rhythm society. Can J Cardiol. 2020;36(6):948-951.

15. Cubeddu LX. Drug-induced inhibition and trafficking disruption of ion channels: pathogenesis of QT abnormalities and drug-induced fatal arrhythmias. Curr Cardiol Rev. 2016;12(2):141-154.

16. Simsek Yavuz S, Unal S. Antiviral treatment of COVID-19. Turk J Med Sci. 2020;50(SI-1):611-619.

17. Million M, Lagier JC, Gautret P, Colson P, Fournier PE, Amrane S, Hocquart M, et al. Early treatment of COVID-19 patients with hydroxychloroquine and azithromycin: A retrospective analysis of 1061 cases in Marseille, France. Travel Med Infect Dis. 2020;35:101738.

18. Geleris J, Sun Y, Platt J, Zucker J, Baldwin M, Hripcsak G, Labella A, et al. Observational study of hydroxychloroquine in hospitalized patients with COVID-19. N Engl J Med. 2020;382(25):2411-2418.
19. Ruiz-Irastorza G, Ramos-Casals M, Brito-Zeron P, Khamashta MA. Clinical efficacy and side effects of antimalarials in systemic lupus erythematosus: a systematic review. Ann Rheum Dis. 2010;69(1):20-28.

20. Chen CY, Wang FL, Lin CC. Chronic hydroxychloroquine use associated with QT prolongation and refractory ventricular arrhythmia. Clin Toxicol (Phila). 2006;44(2):173175.

21. O'Laughlin JP, Mehta PH, Wong BC. Life threatening severe QTc prolongation in patient with systemic lupus erythematosus due to hydroxychloroquine. Case Rep Cardiol. 2016;2016:4626279.

22. Khobragade SB, Gupta P, Gurav P, Chaudhari G, Gatne MM, Shingatgeri VM. Assessment of proarrhythmic activity of chloroquine in in vivo and ex vivo rabbit models. J Pharmacol Pharmacother. 2013;4(2):116-124.

23. Ramalho D, Freitas J. Drug-induced life-threatening arrhythmias and sudden cardiac death: A clinical perspective of long QT, short QT and Brugada syndromes. Rev Port Cardiol. 2018;37(5):435-446.

24. Drew BJ, Ackerman MJ, Funk M, Gibler WB, Kligfield P, Menon V, Philippides GJ, et al. Prevention of torsade de pointes in hospital settings: a scientific statement from the American Heart Association and the American College of Cardiology Foundation. J Am Coll Cardiol. 2010;55(9):934-947.

25. Borba MGS, Val FFA, Sampaio VS, Alexandre MAA, Melo GC, Brito M, Mourao MPG, et al. Effect of high vs low doses of chloroquine diphosphate as adjunctive therapy for patients hospitalized with severe acute respiratory syndrome coronavirus 2 (SARS-CoV-2) infection: a randomized clinical trial. JAMA Netw Open. 2020;3(4):e208857.

26. Yao X, Ye F, Zhang M, Cui C, Huang B, Niu P, Liu X, et al. In vitro antiviral activity and projection of optimized dosing design of hydroxychloroquine for the treatment of severe acute respiratory syndrome coronavirus 2 (SARSCoV-2). Clin Infect Dis. 2020;71(15):732-739.

27. Chorin E, Dai M, Shulman E, Wadhwani L, Bar-Cohen R, Barbhaiya C, Aizer A, et al. The QT interval in patients with COVID-19 treated with hydroxychloroquine and azithromycin. Nat Med. 2020;26(6):808-809.

28. Saleh M, Gabriels J, Chang D, Soo Kim B, Mansoor A, Mahmood E, Makker P, et al. Effect of chloroquine, hydroxychloroquine, and azithromycin on the corrected QT interval in patients with SARS-CoV-2 infection. Circ Arrhythm Electrophysiol. 2020;13(6):e008662.

29. Mercuro NJ, Yen CF, Shim DJ, Maher TR, McCoy CM, Zimetbaum PJ, Gold HS. Risk of QT interval prolongation associated with use of hydroxychloroquine with or without concomitant azithromycin among hospitalized patients testing positive for coronavirus disease 2019 (COVID-19). JAMA Cardiol. 2020.

30. Bessiere F, Roccia H, Deliniere A, Charriere R, Chevalier P, Argaud L, Cour M. Assessment of QT Intervals in a Case Series of Patients With Coronavirus Disease 2019 (COVID-19) Infection Treated With Hydroxychloroquine Alone or in Combination With Azithromycin in an Intensive Care Unit. JAMA Cardiol. 2020. 
31. Lawrence CL, Pollard CE, Hammond TG, Valentin JP. Nonclinical proarrhythmia models: predicting Torsades de Pointes. J Pharmacol Toxicol Methods. 2005;52(1):46-59.

32. Hondeghem LM, Carlsson L, Duker G. Instability and triangulation of the action potential predict serious proarrhythmia, but action potential duration prolongation is antiarrhythmic. Circulation. 2001;103(15):2004-2013.

33. Erikssen G, Liestol K, Gullestad L, Haugaa KH, Bendz B, Amlie JP. The terminal part of the QT interval ( $\mathrm{T}$ peak to $\mathrm{T}$ end): a predictor of mortality after acute myocardial infarction. Ann Noninvasive Electrocardiol. 2012;17(2):8594.

34. Gupta P, Patel C, Patel H, Narayanaswamy S, Malhotra B, Green JT, Yan GX. T(p-e)/QT ratio as an index of arrhythmogenesis. J Electrocardiol. 2008;41(6):567-574.

35. Tse G, Gong M, Meng L, Wong CW, Bazoukis G, Chan MTV, Wong MCS, et al. Predictive value of T peak - T end indices for adverse outcomes in acquired QT prolongation: a meta-analysis. Front Physiol. 2018;9:1226.
36. Topilski I, Rogowski O, Rosso R, Justo D, Copperman Y, Glikson M, Belhassen B, et al. The morphology of the QT interval predicts torsade de pointes during acquired bradyarrhythmias. J Am Coll Cardiol. 2007;49(3):320-328.

37. Yamaguchi M, Shimizu M, Ino H, Terai H, Uchiyama K, Oe K, Mabuchi T, et al. T wave peak-to-end interval and QT dispersion in acquired long QT syndrome: a new index for arrhythmogenicity. Clin Sci (Lond). 2003;105(6):671676.

38. Taubel J, Prasad K, Rosano G, Ferber G, Wibberley H, Cole ST, Van Langenhoven L, et al. Effects of the fluoroquinolones moxifloxacin and levofloxacin on the QT subintervals: sex differences in ventricular repolarization. J Clin Pharmacol. 2020;60(3):400-408.

39. Jankelson L, Karam G, Becker ML, Chinitz LA, Tsai MC. QT prolongation, torsades de pointes, and sudden death with short courses of chloroquine or hydroxychloroquine as used in COVID-19: A systematic review. Heart Rhythm. 2020. 